\title{
A clinical perspective on diagnosis of urinary tract infections in children
}

\author{
Anna Jarvis, MD;* Dennis Scolnik, MD $\dagger$
}

$\mathrm{U}$ rinary tract infections (UTIs) occur relatively frequently throughout childhood, although the exact incidence and prevalence is unknown. During the first year of life, estimated prevalence of bacteriuria is $0.9 \%$ in girls and $2.5 \%$ in boys. After infancy, female prevalence rises to $1.2 \%-1.9 \%$ and male prevalence falls to $0.03 \% .{ }^{1}$ UTIs are uncommon $(0.2 \%-0.4 \%)$ in circumcised boys, but 5 to 20 times more common in uncircumcised boys. ${ }^{2}$ They are also less common in African American than in Caucasian children. $^{3}$ In febrile children from 2 months to 2 years, the prevalence of UTI is approximately $5 \%,{ }^{2}$ and in febrile children, the presence of an upper respiratory infection or otitis media does not rule-out UTI. ${ }^{3}$

UTI presentations vary greatly throughout childhood. In infants, nonspecific complaints of feeding difficulties, anorexia, irritability, vomiting and diarrhea are common. Two-thirds will have a fever, and a few will present with sepsis syndrome or shock. Late onset jaundice, with elevations of direct and indirect bilirubin, may be the only indication of infantile UTI. Toddlers and preschool children may also present in nonspecific fashion, with behaviour changes, vomiting, anorexia, fever, abdominal pain or secondary enuresis. Parents may notice a change in colour, odor or pattern of urination. Frequency, dysuria and urgency are common but not pathognomonic of UTI. School-aged children are more likely to present with "classical" adult symptoms and signs. Note that, in children with recurrent UTIs, complaints may be minimal and findings less obvious than they were with the first infection. If untreated, symptoms of recurrent UTI may subside over 1 to 3 weeks, although the urine culture remains positive.

Costovertebral angle tenderness, rigors and toxicity indicate upper tract involvement, but it is not possible to clinically distinguish lower UTI from pyelonephritis. Studies suggest that $75 \%$ of children under 5 years of age with febrile UTIs have pyelonephritis, and emergency physicians must maintain a high degree of suspicion to avoid missing the diagnosis. The key challenges are: when to send a urine culture, how to obtain the best urine sample, and how to interpret urinalysis and culture results.

\section{When to send a urine culture?}

Cultures are warranted in: a) all febrile infants less than one year of age, b) children with symptoms or signs suggesting UTI, c) children who are toxic, septic, or shocky without obvious cause, d) children with history of recurrent UTI, bladder catheterization or underlying urinary tract anomaly, and e) children with unexplained fever or symptoms.

\section{How to obtain the best urine sample?}

Four options are available.

1. Midstream clean catch sample. This is the least traumatic method. It is appropriate for all children without obvious infection or anomaly of the external genitalia. Lohr and colleages ${ }^{4,5}$ showed that in school-aged children no cleansing is necessary prior to urine collection.

2. Urine bag. The urine bag is used for infants and children who are not toilet trained. Be sure to wash and dry the periurethral area before affixing a bag. Toddlers may void while being cleaned, so be prepared to collect a midstream sample if they do so. Inspect the bag every 10 to 15 minutes. To facilitate observation, make a hole in the front of the diaper and leave the bag dangling outside. Note that bagged specimens are unreliable; therefore, infants and children who will be started on antibiotics prior to urine culture results should not have bagged specimens sent for culture. ${ }^{6}$

3. Catheterization is the method of choice for febrile infants, toxic, septic or shocky children, and for all age groups who require urgent antibiotic treatment. Careful cleansing and strict aseptic technique is imperative to avoid iatrogenic infection. Contraindications include obvious genital infection, labial adhesions and inability to see the ure-

*Professor of Paediatrics, University of Toronto, Division of Emergency Services; Medical Director, Child Health Services, The Hospital for Sick Children, Toronto, Ont.

†Assistant Professor, University of Toronto; Divisions of Emergency Services and Clinical Pharmacology and Toxicology Clinical Pharmacology and Toxicology, The Hospital for Sick Children, Toronto, Ont.

Received: April 10, 2000; accepted: May 5, 2000

This article has been peer reviewed. 
thral opening in uncircumcised boys. The foreskin should never be forcibly retracted as paraphimosis may result.

4. Suprapubic aspiration is reserved for diapered, uncircumcised boys whose urethral opening cannot be visualized and for infants or children who need urgent antibiotic therapy but cannot be catheterized and cannot produce a clean midstream sample. To perform bladder aspiration, first percuss to ensure that urine is present. Then, using sterile technique, insert a 23-gauge, 1-inch needle (attached to a 5-ml syringe) in the midline of the first crease above the pubic bone. Advance the needle slowly, at right angles to the skin surface, with constant suction on the syringe until the sample is obtained. Withdraw, and remove the needle from the syringe before expelling the urine into a sterile container. Bladder aspiration requires technical expertise, and success rates vary from $23 \%-90 \%$. However it is obtained, urine must be promptly analyzed and plated. If there is a delay of more than 30 minutes between collection and plating, the specimen should be refrigerated.

\section{How to interpret urinalysis and culture results?}

\section{White blood cells}

Microscopic visualization of white blood cells or granular casts suggests infection; however, the minimum acceptable cell count and technique of counting remain controversial. Some authors advocate centrifuged urine and a counting chamber, while others use a drop of non-centrifuged urine. More than 5 white blood cells $/ \mathrm{mm}^{3}$ is usually considered indicative of infection, as is a positive leukocyte esterase dipstick test. Of note, Crain and Gershel ${ }^{7}$ showed that more than half the UTIs in infants under 8 weeks of age would have been missed on the basis of a "normal" urinalysis.

\section{The nitrite test}

A positive dipstick test for nitrite is usually accepted as evidence of infection. Unfortunately, bacterial nitrite production takes time; therefore, urine voided shortly after formation may test (falsely) negative, particularly in infants less than 3 months old.?

Table 1. Significant counts for colony forming units on urine culture

\begin{tabular}{lc} 
Specimen & $\begin{array}{c}\text { Significant count } \\
\text { (colony forming units) }\end{array}$ \\
\hline Suprapubic aspiration & $\geq 1 \times 10^{6} / \mathrm{L}$ \\
Cystoscopic, catheterized, MS & $\geq 50 \times 10^{6} / \mathrm{L}$ \\
Bag or unspecified urine & $\geq 100 \times 10^{6} / \mathrm{L}$ \\
\hline
\end{tabular}

MS = midstream clean catch.

Note: Febrile infants who lack clinical indications for hospitalization seldom require intramuscular ceftriaxone prior to culture results.

\section{Culture and colony forming units}

A negative culture on a bag specimen is a reliable indication of the absence of UTI. Because different methods have been utilized to obtain culture specimens, guidelines have been developed ${ }^{2,3}$ to help clinicians interpret colony counts reported by the laboratory (Table 1). Mixed bacterial growth in "clean catch" or bag urine specimens usually reflects contamination; therefore, these methods are contraindicated if the patient's condition mandates immediate antibiotic treatment. Mixed cultures may, however, be significant in children with nonspecific symptoms. In these cases, the child should be reassessed and the urine culture repeated with meticulous attention to collection and transport technique. "Pure growth" must be interpreted in light of the organism, the collection method and the number of colony-forming units reported on culture. ${ }^{2}$ Organisms such as lactobacillus, coagulase negative staphylococci and corynebacterium species are usually dismissed as skin contaminants, and are not considered urinary pathogens in immune competent patients.

\section{Summary}

Urinary tract infections are common throughout childhood and may lead to delayed renal damage. Their presentation varies with age and may be nonspecific; therefore, emergency physicians must vigilant. Urine collection technique and culture interpretation should be based on patient age, history, symptoms, signs and the need for urgent antibiotic therapy.

\section{References}

1. Nash MA, Seigle RL. Urinary tract infections in infants and children. Adv Pediatr Infect Dis 1996;11:403-48.

2. Practice parameter: the diagnosis, treatment and evaluation of the initial urinary tract infection in febrile infants and young children. American Academy of Pediatrics. Committee on Quality Improvement. Subcommittee on Urinary Tract Infection. Pediatrics 1999;103 (4 pt 1):843-52.

3. Shaw KN, Gorelick MH. Urinary tract infection in the pediatric patient. Ped Clin N Am 1999;46:1111-24.

4. Lohr JA, Donowitz LG, Dudley SM. Bacterial contamination rates for non-clean-catch and clean-catch midstream urine collections in boys. J Pediatr 1986;109:659-60.

5. Lohr JA, Donowitz LG, Dudley SM. Bacterial contamination in voided urine collections in girls. J Pediatr 1989;114:91-3.

6. Canadian Paediatric Society Statement. ID 96-01. Management of the febrile one- to 36-month-old child with no focus of infection. Paed Child Health 1996;1:41-5.

7. Crain EF, Gershel JC. Urinary tract infections in febrile infants younger than 8 weeks of age. Pediatrics 1990;86:363-7.

Correspondence to: Dr. Anna Jarvis, 555 University Ave., Toronto ON M5G 1X8, 416 813-5274, anna.jarvis@ sickkids.on.ca; or Dr. Dennis Scolnik, 416 813-5276, dennis.scolnik@sickkids.on.ca 